\title{
Asymmetric muscle function in patients with developmental mandibular asymmetry
}

\author{
Y. DONG*, X. M. WANG ${ }^{\dagger}, \mathrm{M} . \mathrm{Q} . \mathrm{WANG}^{\dagger} \& \mathrm{~S} . \mathrm{E} . \mathrm{WIDMALM}{ }^{\ddagger}{ }^{*}$ Department of Oral Prosthodontics, \\ College of Medicine, Second Affiliated Hospital, Zhe jiang University, Hang zhou, China, ${ }^{\dagger}$ Department of Oral Surgery, College of Stomatology, \\ Fourth Military Medical University, Shaan Xi, China and ${ }^{\ddagger}$ Division of Prosthodontics, Department of Biologic and Materials Science, University \\ of Michigan, Ann Arbor, MI, USA
}

SUMMARY The aim was to test the hypothesis that developmental mandibular asymmetry is associated with increased asymmetry in muscle activity. Patients with mandibular condylar and/or ramus hyperplasia having unilateral cross-bite were compared with healthy subjects with normal occlusion. Muscle activity was recorded with surface electrodes in the masseter, suprahyoid, sternocleidomastoid muscle (SCM) and upper trapezius areas during jaw opening-closing-clenching, head-neck flexionextension, and elevation-lowering of shoulders. Root mean square (RMS) and mean power frequency (MPF) values were calculated and analysed using ANOVA and $t$-tests with $P<0.05$ chosen as significance level. The SCM and masseter muscles showed co-activation during jaw and head movements, significantly more asymmetric in the patients than in the healthy subjects. The RMS and MPF values were higher in the patients than in the controls in the SCM and suprahyoid areas on both sides during jaw opening-closing movement. The results indicate that the ability to perform symmetric jaw and neck muscle activities is disturbed in patients with developmental mandibular asymmetry. This is of clinical interest because asymmetric activity may be an etiologic factor in temporomandibular joint and cervical pain. The results support that co-activation occurs between jaw and neck muscles during voluntary jaw opening and indicate that postural antigravity reflex activity occurs in the masseter area during head extension. Further studies, where EMG recordings are made from the DMA patients at early stages are motivated to verify activity sources and test if the asymmetric activity is associated with muscle and joint pain in the jaw and cervical areas.

KEYWORDS: developmental mandibular asymmetry, electromyography, jaw muscle, neck muscle, muscle dysfunction, power spectrum, cervical pain, posture

Accepted for publication 23 June 2007

\section{Introduction}

Developmental mandibular asymmetry (DMA) has a wide phenotypic expression with varying degrees of craniofacial and dentofacial abnormalities and malformations such as hyperplasia, unilateral cross-bite, occlusal interferences and mandible deviation from the facial midline. Unilateral disk displacement and internal derangement of the temporomandibular joint (TMJ) have been identified as important characteristics associated with mandibular asymmetry $(1,2)$. The opinions differ, however, regarding cause and effect, i.e. does asymmetry dispose for internal TMJ derangement (ID) or is asymmetry secondary to ID? DMA patients with lower facial deformation also suffer from such signs and symptoms as head, neck, shoulder and upper back pain, muscle weakness and abnormal headneck posture more often than compatible groups without DMA (3-6). Those symptoms are often found also in patients with cervical spine disorders (CSD) (7, 8). The reasons for the pain are often difficult to diagnose and the treatment may then only be symptomatic. The functional state of the stomatognathic system is dependant on the mobility of the cervical 
spine and often associated with neck muscle tenderness; factors that are relevant in diagnosis and management of CSD (9).

There is a close functional relationship between the stomatognathic and the head and neck sensory-motor systems. Negative effects of muscle disease and dysfunction $(10,11)$ and positive effects on posture by stomatognathic treatment (12) have been reported. Stomatognathic muscle function is associated with cervical muscle function. Jaw opening is habitually performed, not only by lowering the mandible, but also by simultaneous tilting of the head backwards (13). Studies using electromyographic (EMG) recordings have confirmed the close inter-relationship during simultaneous jaw and head movements, between muscle activity in the stomatognathic system, and between the head- and neck-motor systems (14-17). Co-activation of jaw and neck muscles has also been observed in intramuscular EMG recordings $(18,19)$.

Muscular functional balance and mandibular symmetry are essential both for oral function, mandibular posture, and for head-neck stability (9). Frequency analysis of the EMG recordings may help in understanding muscle function. Analysis of the power spectrum of EMG activity is useful when assessing muscle fatigue (20, 21) and pathology (22). The effects of mandibular deformity on muscular activity are not well-known. Muscle dysfunction and mandibular asymmetry may or may not occur simultaneously. Some patients have mandibular asymmetry without significant neck muscle dysfunction while other patients suffer from such muscle dysfunction without observable head tilting.

The aim of the present study was to test the hypotheses that:

Developmental mandibular asymmetry is associated with increased asymmetry in the left vs. right side EMG activity in masseter-, neck- and shoulder-muscle areas during jaw opening-closing movements, during head flexion-extension movements, and during shoulder elevation-lowering.

\section{Subjects and methods}

Twenty-one patients, diagnosed as having DMA were originally included in the experimental group. Mandibular deformities were not caused by injury or inflammation. Mandibular asymmetry was diagnosed on roentgenograms taken in submental-vertical position (23). The mandible was determined as having lateral deformity when the distance from the mental spine to the half-way point on a line joining the foramina spinosa exceeded $4 \mathrm{~mm}$. Six patients were excluded because they did not come back for the EMG and jaw movement tests. Complete sets of data were recorded from the remaining 15 patients, seven males and eight females, age $23 \cdot 0 \pm 4 \cdot 78$ (s.d.).

All DMA patients had clinically recognizable unilateral mandibular and/or condylar deformity (Fig. 1) and showed pronounced facial asymmetry with cross-bite on the contra lateral side. The mean distance between the mental spine point and the middle line was longer, $12.6 \pm 5.68 \mathrm{~mm}$ (mean \pm s.d.), in the DMA patients than in the control subjects $(1.7 \pm 1.50 \mathrm{~mm}$; $P<0.001)$. Six patients had also partial cross-bite on the hyperplastic side.

Twenty-one healthy dental school students, age $22 \cdot 0 \pm 0.92$ years, 11 men and 10 women, formed the control group. They had normal complete dentitions with over-jet and over-bite ranging from 2 to $5 \mathrm{~mm}$. They had no signs or symptoms of craniocervical or temporomandibular dysfunction/disorders. The subjects were informed about the study procedures and it was explained that surface EMG is noninvasive. Written consent was obtained from all subjects.

A frontal cephalogram was taken as described by Özbek and Köklű (24) from each subject when standing

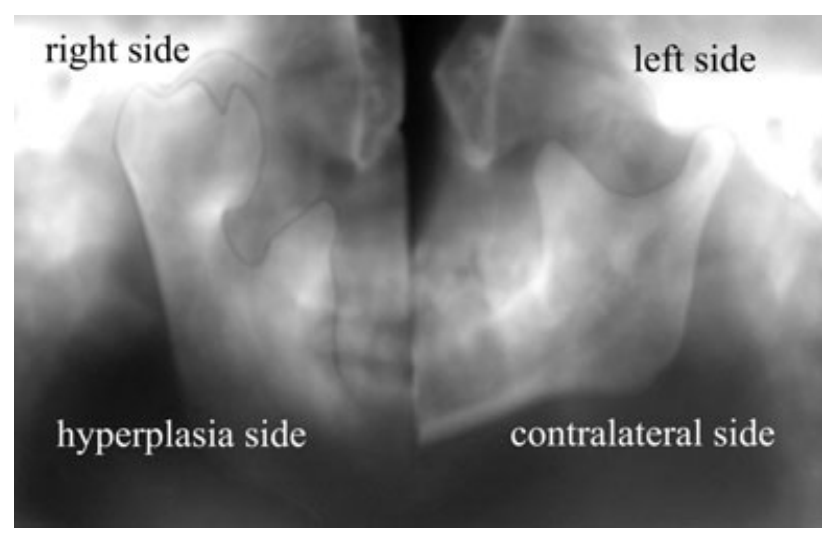

Fig. 1. Tomograms from a DMA patient of central sections through the condylar heads taken with the teeth in centric occlusion. They show how the right condyle and coronoid process were deformed by hyperplasia. An irregular osseous mass with diffuse margins is located around the right condylar head and neck. An artificial joint is formed between the anterior bevel of the articular tubercle and the osseous mass. The bone contours are marked in black. 
erect, looking straight forward, and keeping the head in a natural/upright position. The deviation of a 'cervical line' (from the spinous process of the third to that of the sixth cervical vertebrae) and the cranial central line was measured as described by Pirttiniemi et al. (10) and used as a measure of direction and degree of head tilting.

Mastication and cervical muscle sites were examined for sensitivity to palpation to assess the scope of tenderness for all subjects as described in RDC/TMD (25).

A Sirognathograph (SGG)* and LabVIEW 5.1.1 software $^{\dagger}$ were used for simultaneous recording of jaw movements and EMG to 11 channels. Jaw opening and closing movements were recorded in the frontal and the sagittal planes. The movement graphs (three channels) were used for localization of activity to movement phases and for measurements of jaw deviation in the frontal plane (26). The EMG was recorded simultaneously from eight sites with surface electrodes having a diameter of $10 \mathrm{~mm}^{\ddagger}$. The electrodes were positioned above the centres of the superficial masseter and sternocleidomastoid muscle (SCM) bellies, and in the centre of the suprahyoid area half way between the mental spine and the greater horn of hyoid bone. In the shoulder area the electrodes were positioned above the upper trapezius, $1.5 \mathrm{~cm}$ dorso caudal of the upper margin of the $\mathrm{C} 7$ spinous process. The ground electrode was placed on the right leg. The EMG was amplified and digitized with a 12-bit A/D conversion $\operatorname{card}^{\S}$. The sampling rate was $3 \cdot 1 \mathrm{kHz}$. The high frequency cut off filter was set at $1 \mathrm{kHz}$ with a $0.03 \mathrm{~s}$ time constant. Low frequency cut-off was $2.5 \mathrm{~Hz}$. Data were stored on the hard disk of a Pentium III personal computer**. The surface EMG recording illustrated in Fig. 2 was made for illustrative purpose using the K7 Evaluation System and Duo-trode EMG electrodes ${ }^{\text {II }}$. Scan 11 with a sampling rate of $240 \mathrm{~Hz}$ was used to obtain tracings of processed (integrated) EMG data.

The subjects sat with head unsupported and were asked to maintain a relaxed erect position. EMG activity was recorded during three tasks:

*Siemens Co., Bensheim, Germany.

${ }^{\dagger}$ National Instruments ${ }^{\mathrm{TM}}$, Austin, TX, USA.

${ }^{\ddagger}$ Duotrode; Myotronics Tacoma, Seattle, WA, USA.

§PCL-818 HG; Advantech, Taibei, Taiwan.

**Intel Co., Santa Carla, CA, USA.

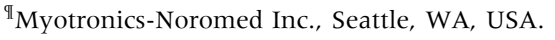

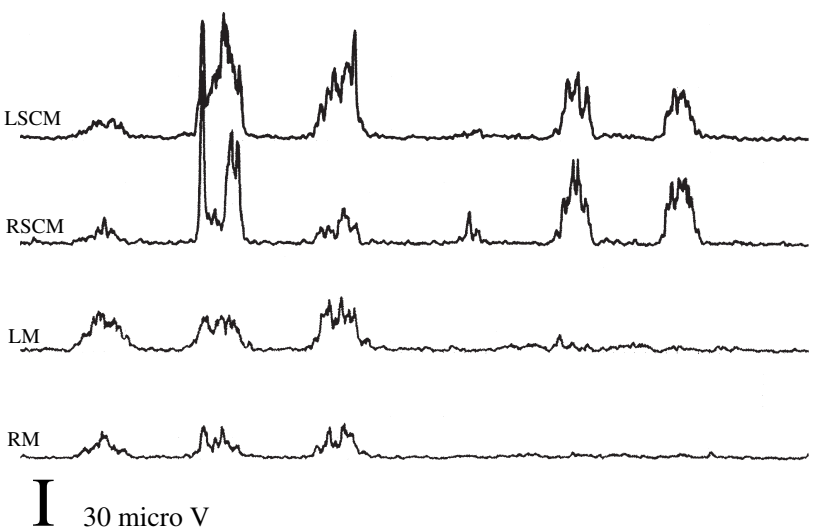

Fig. 2. Tracings of processed (integrated) EMG from a $14 \mathrm{~s}$ long recording made with surface electrodes in a healthy subject from the left SCM (LSCM) and right SCM (RSCM) areas and from the left (LM) and right (RM) masseter muscle areas during three head extensions followed by three head flexions. The masseter activity during head extension was most probably a postural, antigravity reflex with the function to keep the mandible position unchanged relative to the maxilla.

1 Slow (1 per 2 s) self-paced maximal jaw openingclosing movements: Maximal EMG level was first recorded when clenching with maximal force for $3 \mathrm{~s}$ in centric occlusion. The subjects rested for 10 min after the maximal clenching. They then performed jaw opening-closing movements, clenching with ca $10 \%$ of their maximal force for ca $0.5 \mathrm{~s}$ between each movement. The clenching phase was included in the analysis. Subjects were told to open and close in their habitual manner.

2 Head-neck flexion-extension movements (ca 1 per $2 \mathrm{~s})$ : The upper and lower teeth were kept apart with lips sealed and no independent mandibular movements were observed.

3 Shoulder elevation-lowering ( $\mathrm{ca} \mathrm{l} \mathrm{s}^{-1}$ ): Every task was recorded for $10 \mathrm{~s}$ and repeated three times with 3 min rest between the recordings. The recordings were repeated in a second session after 1 week. The EMG and mandibular movement recordings were being accepted as reproducible because two-way ANova for repeated measures did not disclose any significant differences within or between sessions for any subjects for any of the tasks.

\section{Statistical analysis}

A standard fast Fourier algorithm (FFT) with Hanning window was used to convert the EMG signal from the 
time domain to the frequency domain and to produce power spectra with a frequency resolution of $3 \cdot 0 \mathrm{~Hz}$.

Mean power frequency was calculated as:

$$
\mathrm{MPF}=\frac{\int_{0}^{\infty} f \cdot S(f) \mathrm{d} f}{\int_{0}^{\infty} S(f) \mathrm{d} f}
$$

where $f=$ the sampling frequency and $S(f)$ is the EMG power spectral density estimated using an FFT algorithm.

The Root mean square (RMS) energy parameter was computed as:

$$
\mathrm{RMS}=\sqrt{\sum \frac{X_{i}^{2}}{N}}
$$

where $X_{i}=$ the voltage value of sample ${ }_{i}$ and $N=$ total number of samples.

A percentage-overlapping coefficient (POC) (27) was used as muscular functional symmetry parameter. Each contraction was divided into $50 \mathrm{~ms}$ segments. The number of samples varied because the duration of the contractions varied with a mean duration of $0.37 \pm 0.088$ s (s.d.). The RMS values, calculated on the segments were used as variables in the POC formula. POC values were calculated and expressed in $\%$ units using the formula.

$$
\text { POC }=\frac{\left[1-\sum_{i=1}^{j} \mid\left(\text { rightmuscle }_{i}-\text { leftmuscle }_{i}\right) \mid\right]}{\sum_{i=1}^{j}\left(\text { rightmuscle }_{i}+\text { leftmuscle }_{i}\right)} \times 100
$$

A POC of $100 \%$ would indicate perfect symmetry. POC values were then compared between control subjects and DMA patients.

Mean values of RMS values, mean power frequency (MPF) and POC were calculated for each muscle area based on those recordings using a statistical package (sPSS/PC V.10)*. Independent sample $t$-tests were used to test differences between POC values for controls and patients (Table 1). Paired $t$-tests were used to test within subject differences in bilateral EMG recordings (Tables 2 and 3). $P<0.05$ was chosen as significance level. No gender differences were found and that factor was therefore not included in the continued analysis.

\section{Results}

The symmetry values based on the EMG recordings in the masseter, suprahyoid, SCM and upper-trapezius areas (POC \%) were always lower in the DMA group

*SPSS Inc., Chicago, IL, USA.

\begin{tabular}{|c|c|c|}
\hline & $\begin{array}{l}\text { Controls } \\
N=21 \\
\text { Left vs. } \\
\text { right side }\end{array}$ & $\begin{array}{l}\text { Patients } \\
N=15 \\
\text { Contra-lateral vs. } \\
\text { hyperplastic side }\end{array}$ \\
\hline \multicolumn{3}{|l|}{ Jaw open-close-clench } \\
\hline Masseter area & $71 \cdot 2 \pm 5 \cdot 67^{* *}$ & $58 \cdot 3 \pm 15 \cdot 35$ \\
\hline Suprahyoid area & $72 \cdot 4 \pm 4.03 *$ & $63 \cdot 9 \pm 14 \cdot 66$ \\
\hline SCM area & $55 \cdot 4 \pm 8 \cdot 10^{* * *}$ & $33 \cdot 2 \pm 7 \cdot 86$ \\
\hline Upper trapezius area & $52 \cdot 0 \pm 9 \cdot 56^{*}$ & $43 \cdot 2 \pm 12 \cdot 07$ \\
\hline \multicolumn{3}{|l|}{ Head extension-flexion } \\
\hline Masseter area & $62 \cdot 3 \pm 10 \cdot 46^{* * *}$ & $41 \cdot 0 \pm 11 \cdot 89$ \\
\hline Suprahyoid area & $63 \cdot 2 \pm 4.55^{* * *}$ & $53 \cdot 5 \pm 9 \cdot 30$ \\
\hline SCM area & $71 \cdot 5 \pm 5 \cdot 11^{* * *}$ & $46 \cdot 4 \pm 15 \cdot 69$ \\
\hline Upper trapezius area & $62 \cdot 7 \pm 4 \cdot 47^{* * *}$ & $41 \cdot 0 \pm 10 \cdot 63$ \\
\hline \multicolumn{3}{|l|}{$\begin{array}{l}\text { Shoulder } \\
\text { elevation/lowering }\end{array}$} \\
\hline Upper trapezius area & $67 \cdot 9 \pm 5 \cdot 44^{* * *}$ & $48 \cdot 4 \pm 9 \cdot 91$ \\
\hline
\end{tabular}

Table 1. Electromyography of masticatory, neck and shouldermuscles symmetry in control and DMA subjects

Percentage-overlapping coefficient (POC) values (mean and standard deviation) of electromyographic (EMG) bilateral recordings from head, neck and shoulder muscle areas during jaw, head and shoulder movements. The lower the POC values are, the more asymmetric the activity was. The muscle activity was significantly more asymmetrical in the patients than in the control subjects in all muscle areas for all three types of movements. The masseter was always active during head movements but only during the extension phase. The stars indicate significant differences between groups. ${ }^{* * *} P<0.001,{ }^{* *} P<0 \cdot 01,{ }^{*} P<0.05$.

than in the control subjects $(P<0.01)$ during movements and clenching (Table 1).

EMG activity was observed in the suprahyoid areas during head flexion, but not during head extension movements. Simultaneous head-neck movements were observed to always occur both in patients and in control subjects during jaw opening and closing movements. Jaw opening was always accompanied by headneck extension and jaw-closing movement was connected with head-neck flexion in both groups.

EMG contraction activity was always recorded in the masseter during head-extension in spite of no visible independent mandibular movement. That is the subjects did not close the mouth making tooth contact or decrease the free way space. No such EMG activity was observed during head flexion (Fig. 2).

No differences were found in the control group when comparing paired RMS and MPF values between sides for the masseter, the suprahyoid, and the SCM areas during jaw opening-closing-clenching phase, head extensionflexion, or shoulder movements (Tables 2 and 3 ). 
Table 2. RMS values of EMG from control and DMA subjects $(\mathrm{m} v)$

\begin{tabular}{|c|c|c|c|c|c|c|}
\hline & \multicolumn{3}{|l|}{ Control $N=21$} & \multicolumn{3}{|l|}{ Patients $N=15$} \\
\hline & $\begin{array}{l}\text { Right side } \\
\text { (mean } \pm \text { s.d.) }\end{array}$ & $\begin{array}{l}\text { Left side } \\
\text { (mean } \pm \text { s.d.) }\end{array}$ & $\begin{array}{l}\text { Paired differences } \\
\text { (mean } \pm \text { s.e.) }\end{array}$ & $\begin{array}{l}\text { Contra-lateral side } \\
\text { (mean } \pm \text { s.d.) }\end{array}$ & $\begin{array}{l}\text { Hyperplastic side } \\
\text { (mean } \pm \text { s.d.) }\end{array}$ & $\begin{array}{l}\text { Paired differences } \\
\text { (mean } \pm \text { s.e.) }\end{array}$ \\
\hline \multicolumn{7}{|c|}{ Jaw opening-closing-clenching } \\
\hline Masseter area & $16 \cdot 4 \pm 6 \cdot 86$ & $16 \cdot 3 \pm 7 \cdot 43$ & $0 \cdot 1 \pm 0 \cdot 44$ & $13.9 \pm 2.72$ & $10 \cdot 5 \pm 4.53$ & $3 \cdot 3 \pm 1 \cdot 16^{*}$ \\
\hline Suprahyoid area & $9 \cdot 5 \pm 1 \cdot 89$ & $9 \cdot 6 \pm 1.59$ & $-0 \cdot 1 \pm 0 \cdot 34$ & $14.9 \pm 4.32$ & $10 \cdot 7 \pm 4 \cdot 00$ & $4 \cdot 1 \pm 1 \cdot 44^{*}$ \\
\hline SCM & $0 \cdot 8 \pm 0 \cdot 38$ & $0.9 \pm 0.33$ & $-0 \cdot 1 \pm 0 \cdot 04$ & $2 \cdot 9 \pm 0 \cdot 79$ & $3 \cdot 6 \pm 1 \cdot 15$ & $-0 \cdot 7 \pm 0 \cdot 19^{* *}$ \\
\hline Upper trapezius & $0 \cdot 13 \pm 0 \cdot 057$ & $0 \cdot 14 \pm 0 \cdot 063$ & $-0.0 \pm 0.02$ & $0 \cdot 2 \pm 0 \cdot 14$ & $0 \cdot 4 \pm 0 \cdot 16$ & $-0 \cdot 2 \pm 0.05^{* *}$ \\
\hline \multicolumn{7}{|c|}{ Head extension-flexion } \\
\hline Masseter area & $1 \cdot 4 \pm 0 \cdot 39$ & $1 \cdot 4 \pm 0 \cdot 39$ & $-0.0 \pm 0.06$ & $3 \cdot 0 \pm 1 \cdot 18$ & $1 \cdot 4 \pm 0 \cdot 82$ & $1 \cdot 6 \pm 0.33^{* * *}$ \\
\hline Suprahyoid area & $2 \cdot 4 \pm 0 \cdot 61$ & $2 \cdot 4 \pm 0 \cdot 64$ & $-0 \cdot 1 \pm 0 \cdot 07$ & $6 \cdot 5 \pm 2 \cdot 14$ & $3 \cdot 8 \pm 2 \cdot 75$ & $2 \cdot 7 \pm 0 \cdot 79 * *$ \\
\hline SCM & $2 \cdot 0 \pm 0 \cdot 83$ & $2 \cdot 0 \pm 0 \cdot 78$ & $0 \cdot 0 \pm 0 \cdot 05$ & $2 \cdot 1 \pm 0 \cdot 77$ & $3.5 \pm 1.63$ & $-1 \cdot 4 \pm 0.43^{* *}$ \\
\hline Upper trapezius & $0.30 \pm 0.079$ & $0 \cdot 28 \pm 0 \cdot 082$ & $0 \cdot 0 \pm 0 \cdot 01$ & $0 \cdot 3 \pm 0 \cdot 15$ & $0 \cdot 6 \pm 0 \cdot 30$ & $-0 \cdot 3 \pm 0.09^{* *}$ \\
\hline \multicolumn{7}{|l|}{ Shoulder movement } \\
\hline Upper trapezius & $3 \cdot 8 \pm 1 \cdot 69$ & $3 \cdot 5 \pm 2 \cdot 05$ & $0 \cdot 3 \pm 0 \cdot 23$ & $3 \cdot 9 \pm 2 \cdot 15$ & $5 \cdot 8 \pm 2 \cdot 03$ & $1 \cdot 9 \pm 0 \cdot 60^{* *}$ \\
\hline
\end{tabular}

Root mean square (RMS) values (mean and standard deviation) of electromyographic (EMG) bilateral recordings from head, neck, and shoulder muscle areas during jaw, head, and shoulder movements. During head movements masseter activity was observed during head extension but never during head flexion. Significant differences were observed between sides in the patients in all muscle areas during all tasks but never in the control subjects. Note that in the patients the RMS values were lower on the side with hyperplasia in the masseter and suprahyoid areas while they were higher in the neck and shoulder areas. The stars indicate significant differences between sides. $* * * P<0.001, * * P<0.01, * P<0 \cdot 05$.

Table 3. Frequency values of EMG from controland DMA subjects $(\mathrm{Hz})$

\begin{tabular}{|c|c|c|c|c|c|c|}
\hline & \multicolumn{3}{|l|}{ Control $N=21$} & \multicolumn{3}{|l|}{ Patients $N=15$} \\
\hline & $\begin{array}{l}\text { Right side } \\
\text { (mean } \pm \text { s.d.) }\end{array}$ & $\begin{array}{l}\text { Left side } \\
\text { (mean } \pm \text { s.d.) }\end{array}$ & $\begin{array}{l}\text { Paired differences } \\
\text { (mean } \pm \text { s.e.) }\end{array}$ & $\begin{array}{l}\text { Contra-lateral side } \\
\text { (mean } \pm \text { s.d.) }\end{array}$ & $\begin{array}{l}\text { Hyperplastic side } \\
\text { (mean } \pm \text { s.d.) }\end{array}$ & $\begin{array}{l}\text { Paired differences } \\
\text { (mean } \pm \text { s.e.) }\end{array}$ \\
\hline \multicolumn{7}{|c|}{ Jaw opening-closing-clenching } \\
\hline Masseter area & $188 \pm 76 \cdot 3$ & $193 \pm 78 \cdot 2$ & $-4 \cdot 7 \pm 5 \cdot 63$ & $180 \pm 38 \cdot 9$ & $210 \pm 40 \cdot 7$ & $-29 \cdot 9 \pm 10 \cdot 87^{*}$ \\
\hline Suprahyoid area & $224 \pm 43 \cdot 2$ & $221 \pm 42 \cdot 4$ & $3 \cdot 6 \pm 4 \cdot 47$ & $279 \pm 68 \cdot 4$ & $286 \pm 67 \cdot 4$ & $-7 \cdot 1 \pm 2 \cdot 81^{*}$ \\
\hline SCM & $216 \pm 59 \cdot 7$ & $218 \pm 75 \cdot 0$ & $-2 \cdot 8 \pm 8 \cdot 34$ & $253 \pm 62 \cdot 0$ & $245 \pm 63 \cdot 3$ & $7 \cdot 5 \pm 3 \cdot 22^{*}$ \\
\hline Upper trapezius & $229 \pm 41 \cdot 0$ & $226 \pm 36 \cdot 5$ & $2 \cdot 8 \pm 8 \cdot 80$ & $257 \pm 45 \cdot 1$ & $248 \pm 45 \cdot 5$ & $9 \cdot 0 \pm 11 \cdot 24$ \\
\hline \multicolumn{7}{|c|}{ Head extension-flexion } \\
\hline Masseter area & $219 \pm 73 \cdot 3$ & $218 \pm 70 \cdot 1$ & $-0 \cdot 6 \pm 3 \cdot 27$ & $189 \pm 72 \cdot 6$ & $196 \pm 75 \cdot 6$ & $-7 \cdot 6 \pm 3 \cdot 52 *$ \\
\hline Suprahyoid area & $183 \pm 52 \cdot 9$ & $184 \pm 54 \cdot 0$ & $0 \cdot 8 \pm 1 \cdot 75$ & $193 \pm 54 \cdot 0$ & $204 \pm 51 \cdot 1$ & $-10 \cdot 4 \pm 3 \cdot 47^{*}$ \\
\hline SCM & $181 \pm 46 \cdot 9$ & $176 \pm 44 \cdot 7$ & $-5 \cdot 2 \pm 5 \cdot 72$ & $221 \pm 36 \cdot 0$ & $211 \pm 25 \cdot 4$ & $10 \cdot 1 \pm 4 \cdot 08^{*}$ \\
\hline Upper trapezius & $151 \pm 39 \cdot 8$ & $155 \pm 36 \cdot 7$ & $4 \cdot 3 \pm 7 \cdot 78$ & $180 \pm 34 \cdot 5$ & $164 \pm 35 \cdot 9$ & $15 \cdot 7 \pm 3 \cdot 27^{* * *}$ \\
\hline \multicolumn{7}{|l|}{ Shoulder movement } \\
\hline Upper trapezius & $170 \pm 38 \cdot 4$ & $172 \pm 46 \cdot 9$ & $-1 \cdot 3 \pm 2 \cdot 72$ & $204 \pm 37 \cdot 6$ & $182 \pm 20 \cdot 0$ & $21 \cdot 8 \pm 7 \cdot 20^{* *}$ \\
\hline
\end{tabular}

Mean power frequency (MPF) values (mean and standard deviation) of electromyographic (EMG) bilateral recordings from head, neck and shoulder muscle areas during jaw, head and shoulder movements. Significant differences (paired samples $t$-test) were observed between eight of nine areas in the patients but in none in the control subjects. Note that in the patients the MPF values were higher on the side with hyperplasia in the masseter and suprahyoid areas while they were lower in the neck and shoulder areas. No such differences were found in the control subjects. The stars indicate significant differences between sides. ${ }^{* *} P<0 \cdot 001,{ }^{* *} P<0 \cdot 01,{ }^{*} P<0 \cdot 05$.

In the patient group, the RMS values were lower on the hyperplastic side in the masseter and suprahyoid areas and higher in the SCM and upper trapezius areas during all three types of movements (Table 2).
The frequency distribution was more asymmetric in the patient than in the control group. The masseter and the suprahyoid power frequency (MPF) values were higher but the SCM and trapezius MPF values were 
lower on the side affected with hyperplasia during jaw and head movements (Table 3).

The frequency distribution in the EMG recordings from the suprahyoid, SCM and upper trapezius areas was shifted to a higher area in the DMA patients than in the control subjects (Table 3; $P<0 \cdot 05$ ).

There were easily observable mandibular deviations during jaw opening-closing movements in all DMA patients and they were always to the hyperplastic side. Mean maximum lateral deviation was $0.7 \pm 0.66 \mathrm{~mm}$ for the left side, $0.7 \pm 0.79 \mathrm{~mm}$ for the right side in control group, while $2.6 \pm 2.33$ and $3.3 \pm 1.00 \mathrm{~mm}$ to contra-lateral side and hyperplastic side in patient group.

All patients had the head tilted to the non-hyperplastic side while standing erect in a habitual upright position. While slight deviations and tilting occurred in the control group, no preferences for side were observed.

Pain grade and scope was more on the hyperplastic side than on the unaffected side $(P<0.05)$ at muscle palpation in the masseter and neck areas in patients (Nonparameter tests-Wilcoxon Signed Ranks Test).

\section{Discussion}

Although healthy, no subjects should be expected to have bilaterally symmetric activity close to $100 \%$ because facial skeletons are seldom, if ever, perfectly symmetrical. The result of the present study was that the patients with developmental mandibular asymmetry (DMA) had more asymmetrical EMG activity in the masseter, suprahyoid, SCM and upper-trapezius areas during jaw, head, neck and shoulders movements and clenching than the healthy control subjects. It was observed that the DMA patients' heads were tilted to the affected side when standing in their habitual upright position. The patients had unilateral hyperplasia and they all had mandibular deviations to the unaffected side during jaw movements. The primary cause for the pronounced mandibular asymmetry was in these patients most probably their congenital disease DMA, which is known to cause condylar and/or condylar neck hyperplasia. Some authors have suggested internal TMJ derangement (ID) as a possible cause of condylar growth changes and mandibular asymmetry $(1,28)$ while others have suggested ID as secondary to mandibular asymmetry (2). This uncertainty and a similar one about muscular dysfunction being primary or secondary to skeletal asymmetry and changed head and neck posture make it of high interest to follow DMA patients from an early stage. This will be considered in future studies. Asymmetrical activity may be an aetiological factor causing changes in the cervical spine curvature and facial muscular pain. The findings are of interest when discussing postural abnormalities, diagnosis and treatment plans in patients with TMD and cervical pain.

Our results confirm that co-activation of jaw and neck muscles occur during jaw opening-closing movements $(14,16)$. We observed that head-neck extension-flexion movements occurred regularly during jaw opening-closing movements. Masseter and suprahyoid muscle areas were active also during head-neck movements both in the control and in the patient groups.

Muscles are stretched during joint movements. Antigravity muscles exhibit static stretch reflexes and have what is called postural contraction or tone. Such tone may also be present in muscles, which are not strictly postural. A muscle can, however, be so relaxed that no electrical or mechanical activity can be observed. The remaining tone it then a result of its inherent elasticity and not of neurological mechanisms (29). When our subjects were sitting in an upright comfortable position the masseters were silent. Contractions were, however, always observed during head extension (Fig. 2), which was performed without observable independent mandibular movement. This activity was most probably a postural, antigravity reflex with the purpose to keep the mandible's position unchanged relative to the maxilla during the head movement. We recorded during movements while Forsberg et al. (30) recorded masseter activity during fixed, static positions. They found only very low activity during 'natural head position' but increased activity was recorded during 5, 10 and 20 degrees of extension. While not directly comparable we consider our results to support those reported by Forsberg et al.

Many clinical studies have observed that patients with craniofacial pain often have pain in craniocervical structures. Neck pain has been shown to be associated with temporomandibular symptoms in general and particularly with facial and jaw pain (31). An attempt to relieve neck symptoms by ergonomic therapy was efficient but only if the masticatory system was also treated $(8,9)$. Associations between neck pain and oral parafunctional muscle activities have been found already in pre-school children $(32,33)$. The aetiology 
of co-existing craniomandibular and cervical spinal pain is most probably multi factorial. It is now widely accepted that the fusimotor system plays a significant role in the mechanisms of such co-existing craniomandibular and cervical spine pain $(7,8)$.

The MPF during muscle contraction is related to the number of synchronized motor units and action potential conduction velocity. A shift of MPF to lower areas is considered to indicate muscular fatigue $(21$, 22 ). The power spectrum obtained from muscles of patients with myogenic pathologies is known to shift towards higher frequencies, while a shift towards lower frequencies is characteristic of muscles in patients with neurogenic diseases (34). Sometime, neurogenic disorders such as oral mandibular dystonia can cause spastic muscle activity, similar to what we observed in our DMA patients and characterized by involuntary movements that affect posture (35). We did, however, not observe any other signs that could justify a diagnosis of a neurogenic disease in any of our DMA patients.

Eleven of the 15 DMA patients in our study had pain, and the pain was more often on the hyperplastic than on the unaffected side. These observations, which need confirmation in future studies, may be related to the abnormal condylar structure or increased muscle tension caused by mandibular ramus hyperplasia. Muscular pain on the hyperplastic side was combined with lower EMG activity (lower RMS values) indicating that the muscles were prone to fatigue. Our DMA patients had unilateral condylar hyperplasia, which limited their condylar/mandibular movement range (Fig. 3). We recorded the mandibular range of motion during jaw open-close movement and deviations in the front plane were always large and to the hyperplastic side in the patient group (Fig. 4). This indicates that unilaterally reduced mobility explains the asymmetric activity.

RMS and MPF values were higher in the patients than in the controls in the SCM and suprahyoid areas on both sides during jaw opening-closing movements. Increased tension may have been needed to stabilize the head position during mandible movement.

Hypertonia is usually found in connection with disorders of the descending pathways that result in decreased inhibitory influence on the motor neurons (36). It is possible that such mechanisms explain the observed hyperactivity in the patients' neck muscles but the activity may also be a functional adaptation to
Fig. 3. TMJ Scholler position. The movement range of the condyle on the hyperplastic side (a) was limited during maximal jaw opening movements while the movement range of the contralateral condyle (b) was normal. The part with deformity is not visible in these radiograms. Outlines are marked with black lines.
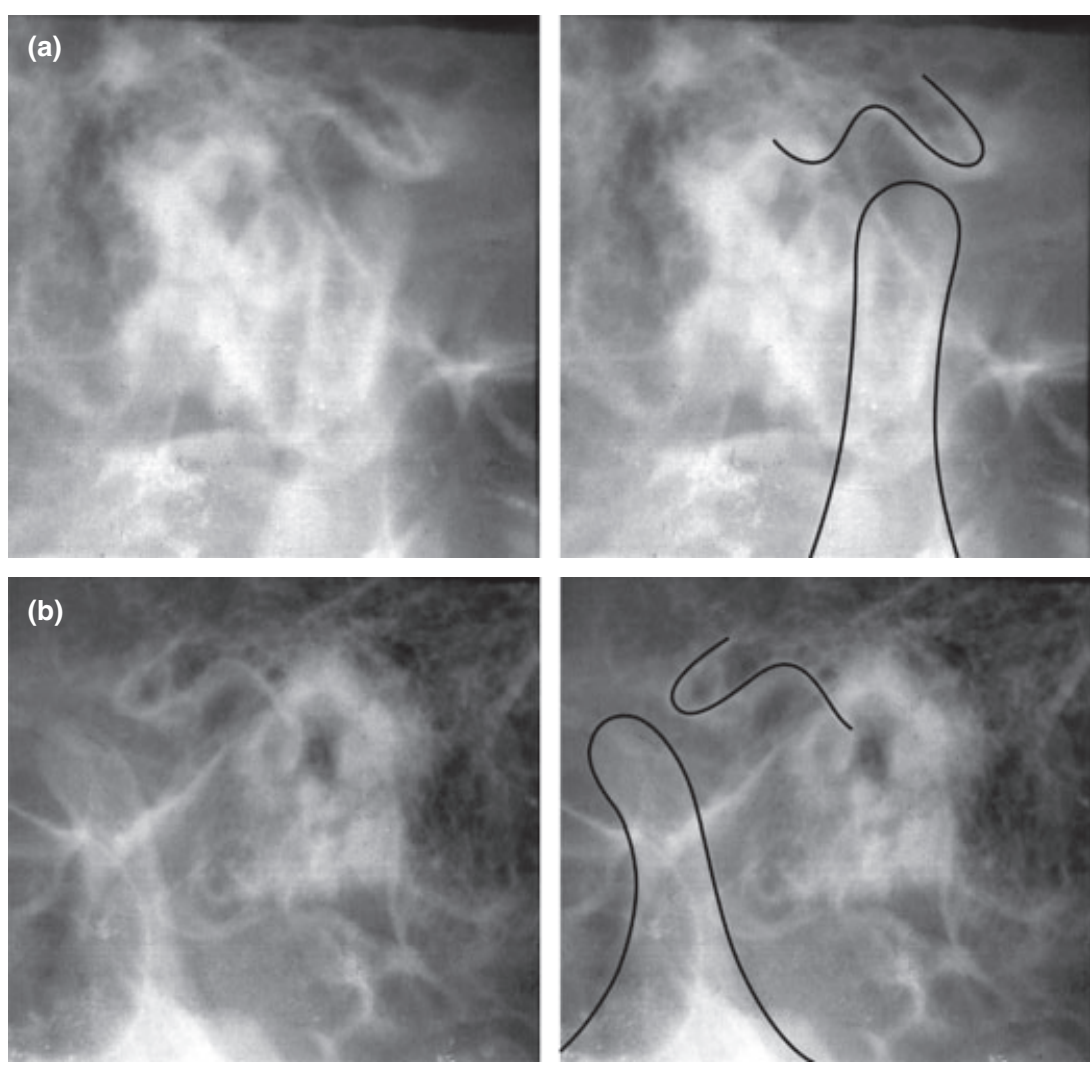
(a)

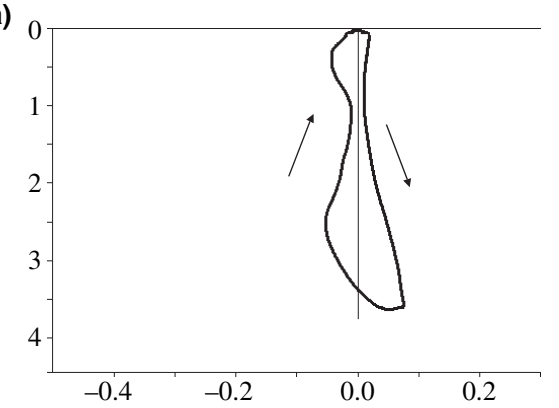

(b)

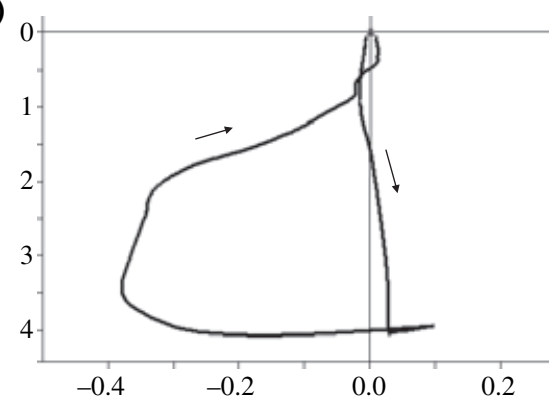

Fig. 4. Average cycle graphs were made using the Sirognathograph to illustrate the differences between the controls (a) and the DMA patients (b) in deviation pattern in the frontal plane during large opening-closing movements. There was marked deviation of the trajectory the incision travels to the hyperplastic side in all DMA patients while deviations were smaller and not unilateral in the control group. Scales are in $\mathrm{cm}$. Negative figures mark magnitude of deviation to the left.

the abnormal cervical posture (Fig. 5a,b) caused by the disease.

The thickness of the subcutaneous tissues on EMG recordings is a well-known factor that affects the amplitude of surface EMG recordings. In clinic, we found the masseter area on the hyperplastic side to look 'thinner' than on the opposite side in the DMA patient. We could not know if this was because of differences in muscle volume or in thickness of subcutaneous tissues. Nor did we have any method for measuring the thickness of the subcutaneous tissue layer. Such measurement can be made in intramuscular EMG recordings by observing how deep the needle has to penetrate before activity is recorded. Such estimates will be considered in future experiments (37).

Morphologic and functional differences between sides are common in body-paired structures. Obviously, the tilted cervical spine changes how gravity influences the neck muscles' activity. Muscular functional balance is essential both for oral function and for head-neck stability (9). The cervical part of the spine is

(a)

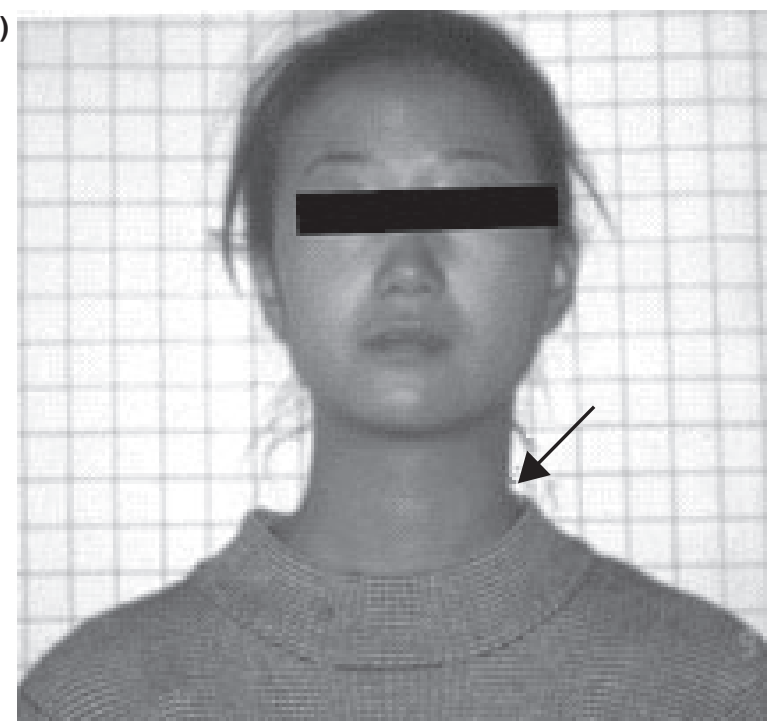

(b)

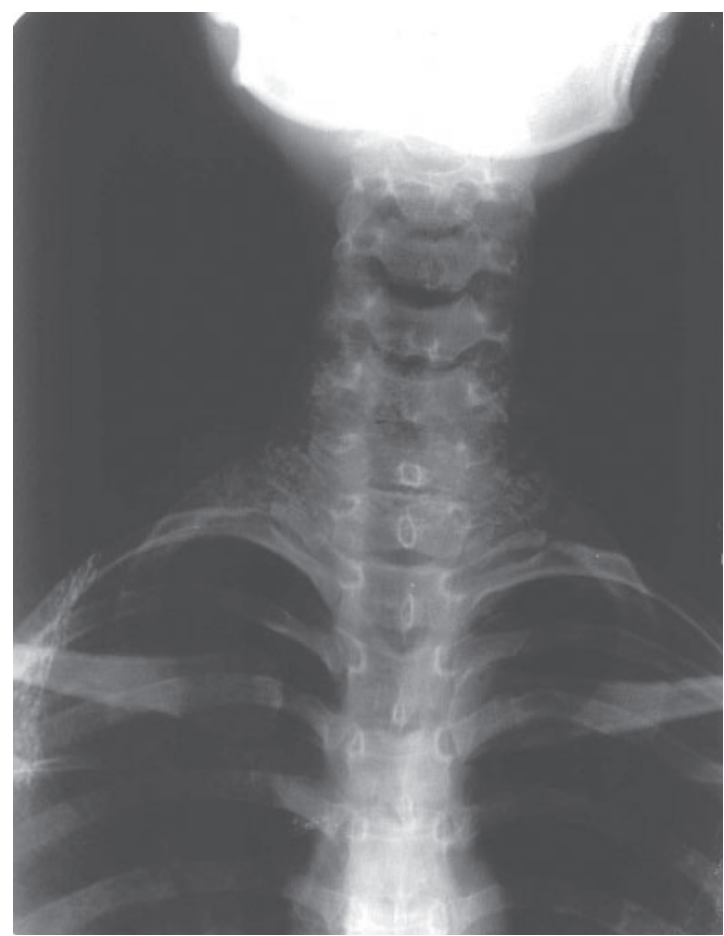

Fig. 5. (a) The figure shows a DMA patient with typical facial asymmetry. The mandibular midline deviates, and the head-neck are tilted during habitual head posture. Hypertonic tension is visible in the sternocleidomastoid muscle (SCM) area on the left side (arrow). It is not possible to know if the curved spine caused tension in the SCM or if a hyperactive SCM caused the tilting. Further studies may clarify this point by recording at initial stages of DMA. Note the raised left shoulder. (b) Roentgenogram in posterior-anterior position of the cervical spine from the DMA patient in (a) showing that the cervical spine is curved and the head is tilted. Such deviation was always to the side with muscle hyperactivity in SCM. 
an unstable segment of the spine. Unbalanced load may increase the instability. In our present study, both the masticatory and the head-neck areas displayed a more pronounced asymmetry in the EMG activity in DMA subjects than in the control subjects. Therefore, DMA may be a factor in cervical spine degeneration, causing head-neck pain, and an abnormal spine curvature.

\section{Conclusion}

In conclusion, the results showed that the facial asymmetry in DMA patients is associated with EMG patterns during jaw, head and shoulders movements that are more asymmetric than in healthy subjects. Such asymmetric muscle function may be an etiologic factor causing cervical pain in DMA patients, which will be considered and tested in future studies. The results also showed antigravity muscle activity in the masseter area during head extension and support the concept of a functional relationship between the temporomandibular system and the cervical spine neuromuscular system.

\section{Acknowledgments}

The authors thank Laura Klem, Senior Research Associate, Center for Statistical Consultation and Research, The University of Michigan, for advice regarding statistical analysis. The authors acknowledge Prof. Zhang Tongsheng, Department of Neurology, University of New Mexico, School of Medicine, for his assistance, technical support and comments on the manuscript. We thank the Chinese People's Liberation Army for funding of the project.

\section{References}

1. Westesson PL, Tallents RH, Katzberg RW, Guay JA. Radiographic assessment of asymmetry of the mandible. Am J Neuroradiol. 1994;15:991-999.

2. Inui M, Fushima K, Sato S. Facial asymmetry in temporomandibular joint disorders. J Oral Rehabil. 1999;26:402-406.

3. Williamson EH, Simmons MD. Mandibular asymmetry and its relation to pain dysfunction. Am J Orthod. 1979;76:612617.

4. Bezuur JN, Habets LL, Hansson TL. The recognition of craniomandibular disorders; condylar symmetry in relation to myogenous and arthrogenous origin of pain. J Oral Rehabil. 1989; 16:257-260.

5. Miyatake E, Miyawaki S, Morishige Y, Nishiyama A, Sasaki A, Takano-Yamamoto T. Class III malocclusion with severe facial asymmetry, unilateral posterior crossbite, and temporomandibular disorders. Am J Orthod Dentofacial Orthop. 2003;124:435-445.

6. Catanzariti JF, Debuse T, Duquesnoy B. Chronic neck pain and masticatory dysfunction. Joint Bone Spine. 2005;72:515519.

7. Browne PA, Clark GT, Kuboki T, Adachi NY. Concurrent cervical and craniofacial pain. Oral Surg Oral Med Oral Pathol Oral Radiol Endod. 1998;86:633-640.

8. Visscher CM, Lobbezoo F, de Boer W, van der Zaag J, Naeije $M$. Prevalence of cervical spine pain in craniomandibular pain patients. Eur J Oral Sci. 2001;109:76-80.

9. Kirveskari P, Alanen P, Karskela V, Kaitaniemi P, Holtari M, Virtanen $\mathrm{T}$ et al. Association of functional state of stomatognathic system with mobility of cervical spine and neck muscle tenderness. Acta Odontol Scand. 1988;46:281-286.

10. Pirttiniemi P, Lahtela P, Huggare J, Serlo W. Head posture and dentofacial asymmetries in surgically treated muscular torticollis patient. Acta Odontol Scand. 1989;47:193-197.

11. Huggare J, Raustia AM. Head posture and cervicovertebral and craniofacial morphology in patients with craniomandibular dysfunction. J Craniomandib Pract. 1992;10:173-179.

12. Huggare J, Pirttiniemi P, Serlo W. Head posture and dentofacial morphology in subject treated for scoliosis. Proc Finn Dent Soc. 1991;87:151-158.

13. Bauer F Are opening movements restricted to movements performed by the lower jaw? [Udfœres en gabebevægelse udelukkende som en sænkning av underkæben?] Tandlægebladet. 1964; 68: 423-428. (In Danish with English summary).

14. Strenge H, Jahns R. Activation and suppression of the trapezius muscle induced by transcranial magnetic stimulation. Electromyogr Clin Neurophysiol. 1998;38:141-145.

15. Eriksson PO, Zafar H, Nordh E. Concomitant mandibular and head-neck movements during jaw opening-closing in man. J Oral Rehabil. 1998;25:859-870.

16. Eriksson PO, Häggman-Henrikson B, Nordh E, Zafar H. Co-ordinated mandibular and head-neck movements during rhythmic jaw activities in man. J Dent Res. 2000;79:1378-1384.

17. Zafar H, Nordh E, Eriksson PO. Temporal coordination between mandibular and head-neck movement during jaw openingclosing tasks in man. Arch Oral Biol. 2000;45:675-682.

18. Kerr FW. Central relationships of trigeminal and cervical primary afferent in the spine cord and medulla. Brain Res. 1972;43:561-572.

19. Widmalm SE, Lillie JH, Ash MM Jr. Anatomical and electromyo - graphic studies of the digastric muscle. J Oral Rehabil. 1988;15:3-21.

20. Lindström L, Magnusson R, Petersén I. Muscular fatigue and action potential conduction velocity changes studied with frequency analysis of EMG signals. Electromyography. 1970;10:341-356.

21. Gay T, Maton B, Rendell J, Majourau A. Characteristics of muscle fatigue in patient with myofascial pain-dysfunction syndrome. Arch Oral Biol. 1994;39:847-852.

22. Cywińska-Wasilewska G, Ober JJ, Koczocik-Przedpelska J. Power spectrum of the surface EMG in post-polio syndrome. Electromyogr Clin Neurophysiol. 1998;38:463-466. 
23. Forsberg CT, Burstone CJ, Hanley KJ. Diagnosis and treatment planning of skeletal asymmetry with the submental-vertical radiograph. Am J Orthod. 1984;85:224-237.

24. Özbek MM, Köklü A. Natural cervical inclination and craniofacial structure. Am J Orthod Dentofacial Orthop. 1993;104: 584-591.

25. Dworkin SF, LeResche L. Research diagnostic criteria for temporomandibular disorders. J Craniomandib Disord. 1992;6:301-355.

26. Arthur L. Electrognathographics: Atlas of Diagnostic Procedures and Inter-Pretation. Chicago, IL: Quintessence Publishing Co., Inc.; 1985: 61.

27. Ferrario VF, Sforza C, Colombo A, Ciusa V. An electromyographic investigation of masticatory muscles symmetry in normo-occlusion subjects. J Oral Rehabil. 2000;27:3340.

28. Ahn SJ, Lee SP, Nahm DS. Relationship between temporomandibular joint internal derangement and facial asymmetry in women. Am J Orthod Dentofacial Orthop. 2005;128:583591.

29. Gardner E. Fundamentels of Neurology a Psychophysiological Approach. 6th edn. Philadelphia, PA: WB Saunders; 1975: 117-118.

30. Forsberg CM, Hellsing E, Linder-Aronson S, Sheikholeslam A. Emg activity in neck and masticatory muscles in relation to extension and flexion of the head. Eur J Orthod. 1985;7:177184.

31. Ciancaglini R, Testa M, Radaelli G. Association of neck pain with symptoms of temporomandibular dysfunction in the general adult population. Scand J Rehabil Med. 1999;31:1722.

32. Widmalm SE, Christiansen RL, Gunn SM, Hawley LM. Prevalence of signs and symptoms of cranio mandibular disorders and of orofacial parafunction in 4-6 year old African-American and Caucasian children. J Oral Rehabil. 1995;22:87-93.

33. Widmalm SE, Gunn SM, Christiansen RL. Oral parafunctions as TMD risk factors in children. J Craniomandib Pract. 1995; 13:242-246.

34. Bilodeau M, Arsenault AB, Gravel D, Bourbonnais D. EMG power spectrum of elbow extensors: a reliability study. Electromyogr Clin Neurophysiol. 1994;34:149-158.

35. Laskawi R, Rohrbach S. Oromandibuläre Dystonien. Laryngol Rhinol Otol. 2001;80:708-713.

36. Vander A, Sherman J, Luciano D. Human Physiology. The Mechanisms of Body Function. 8th edn. Boston: McGraw Hill; 2001: 346-347.

37. Van der Glas HW, Lobbezoo F, van-der-Bilt A, Bosman F. Influence of the thickness of soft tissues overlying human masseter and temporalis muscles on the electromyographic maximal voluntary contraction level. Eur J Oral Sci. 1996; 104:87-95.

Correspondence: Dr Dong Yan, DDS, PhD, Department of Oral Prosthodontics, College of Medicine, Second affiliated hospital, Zhe jiang University. 1, Jie Fang Road, Hang zhou, China. Tel.: +571 87784619; Fax: +571 87022776;

E-mail: wenqing66_@yahoo.com.cn 